\title{
Plasma excitations of dressed Dirac electrons in
}

\section{graphene layers}

\author{
Oleksiy Roslyak, ${ }^{* \dagger}$ Godfrey Gumbs ${ }^{\dagger}$ and Danhong Huang \\ Department of Physics and Astronomy, Hunter College at the City University of New York, 695 \\ Park Avenue New York, NY 10065, USA, and Air Force Research Laboratory, Space Vehicles \\ Directorate, Kirtland Air Force Base, NM 87117, USA \\ E-mail: avroslyak@gmail.com
}

\begin{abstract}
Collective plasma excitations of optically dressed Dirac electrons in single and double graphene layers are calculated in the RPA approximation. The presence of circularly polarized light gives rise to an energy gap $E_{g}$ between the conduction and valence energy bands. Its value may be adjusted by varying the frequency and intensity of the light, and may reach values of the gap reported for epitaxially grown graphene and far exceeding that caused by spinorbit coupling. We report plasmon dispersion relations for various energy gaps and separation between graphene layers. For a single graphene sheet, we find that plasmon modes may be excited for larger wave vector and frequency when subjected to light. For double layers, we obtained an optical and phonon-like mode and found that the optical mode is not as sensitive as the phonon-like mode in the long wavelength limit when the layer separation is varied, for a chosen $E_{g}$. The dressed electron plasma although massive still has Dirac origin giving rise to anomalous plasmon behavior upon crossing the $\omega=\hbar v_{F} q$ cone.
\end{abstract}

\footnotetext{
*To whom correspondence should be addressed

${ }^{\dagger}$ Department of Physics and Astronomy, Hunter College at the City University of New York, 695 Park Avenue New York, NY 10065, USA

$\ddagger$ Air Force Research Laboratory, Space Vehicles Directorate, Kirtland Air Force Base, NM 87117, USA
} 


\section{Introduction}

Recently, several groups have addressed the effects which a circularly polarized electromagnetic field $(\mathrm{CPEF}){ }^{1}{ }^{1}$ spin-orbit interaction $(\mathrm{SOI})^{[2}$ in suspended graphene or the sub-lattice symmetry breaking (SSB) by an underlying polar substrate ${ }^{3-5}$ in epitaxial graphene may have on the energy band structure of a graphene sheet. In all cases, a gap is opened between the valence and conduction bands as well as between the intra-band and inter-band electron-hole excitation continuum of the otherwise semi-metal Dirac system.6-9 As a matter of fact, the interplay between the singleparticle excitations in the long wavelength limit results in dielectric screening of the Coulomb interaction which produces an undamped plasmon mode that appears in the gap separating the two types of electron-hole modes forming a continuum. By this we mean that a self-sustained collective plasma mode is not supported by exciting either the intra-band or inter-band single-particle modes only. Furthermore, although the Dirac electrons near the K-points now acquire a non-zero effective mass, one still cannot produce a long wavelength plasmon mode as in the two-dimensional electron gas (2DEG) by intra-band excitations for intrinsic graphene.

Plasmon excitations in semi-metallic graphene were studied extensively theoretically in single and multilayer graphene. However, their experimental observations are limited so far to either inelastic scattering of highly energetic particles (faster than $10^{5} \mathrm{~m} / \mathrm{s}$ ) or indirect observation through plasmoritons (a quasiparticle formed by resonant coupling of renormalized hole states with the plasmons). Plasmons in graphene gapped by SOI or by a polar substrate have been investigated by Wang and Chakraborty ${ }^{2]}$ as well as Pyatkovskiy ${ }^{[3}$ Because the SOI is small $(\sim 0.05 \mathrm{meV})$, its effect on dielectric screening of the Coulomb interaction requires either high resolution spectroscopy or low electron density in the conduction band to be observed. The effect of SOI on the plasmon excitations in a 2DEG were also studied by several authors $\frac{10-12}{-12}$ On the other hand, the gap between the valence and conduction bands near the Fermi level when circularly polarized light is applied to graphene could be manipulated, unlike the intrinsic gap due to SOI, depending on the intensity and frequency of the light. For a $\mathrm{CO}_{2}$ laser with a $70 \mathrm{~W}$ output power and wavelength of $10 \mu$, the energy gap is about $100 \mathrm{meV}$, compared with $0.08 \mathrm{meV}$ induced by SOI.$^{-2}$ The exact gap 
opening mechanism in epitaxial graphene is still under debate. ${ }^{13}$ The problem is rather technical and arises from the fact that the graphene sample sits on top of a buffer layer which provides an additional mid gap level, thus obscuring the exact energy dispersion curve and requires numerical $a b$ initio calculations $\frac{14}{14}$ This ambiguity stimulated discussion regarding the symmetry breaking gap ${ }^{15}$ versus the effects due to electron-electron interaction. ${ }^{16}$ This also not only indicates the importance of theoretical investigation of plasmon in gapped graphene, but also an alternative means of providing the gap. Since SOI is too weak to observe its effect, dressing of the Dirac electrons with CPEF is seems to be an alternative worthy of further investigation.

The value of this dynamic energy gap is comparable with that induced by a polar substrate, but contrary to the latter, it is highly adjustable. As a matter of fact, those two gap-opening mechanisms may even work conjunctively. In that case, the circular polarization of the electromagnetic field may no longer be required provided that it is tuned to be in resonance with the SSB gap. A possible experiment to measure the plasmon frequencies in single and double layer graphene could employ electron energy loss spectroscopy (EELS), $17-19$ as shown schematically in Fig.[figure][1][]1. Essentially, this would be a pump-probe, with the electron beam being the probe effected by the polarized light whose role is to open the gap. The Raman shift of the scattered electrons provides both particle-hole and plasmon excitation frequencies, which are usually characterized by their spectral weight, a quantity that depends on the transferred energy $\hbar \omega$ and momentum $\hbar q$.

The remainder of this paper is organized as follows. In Sec. II, we present the model Hamiltonian for dressed Dirac electrons by a CPEF as well as corresponding energy eigenvalues and wave-functions. These results are then used for calculating the polarization function and plasmon dispersion relation in the RPA. Our numerical results are presented in Sec. III but, for convenience, we briefly summarize the highlights of our calculations. For a single graphene layer, in the presence of CPEF, we calculate analytically the real and imaginary parts of the noninteracting polarization function, whose imaginary part provides the particle-hole spectral weight. As a piecewise continuous function, it is expressed in terms of region functions which provide a convenient way for determining the Landau damping of the plasmon excitations. By increasing the intensity of CPEF, 
the Dirac electrons become more dressed by photons, providing them with a larger and larger effective mass. Regions in $\omega-q$ space, which are non-existent for conventional Dirac electrons, emerge thereby making the plasmon behavior to resemble that of conventional 2DEG. However, the resemblance is limited, and we show there is no complete crossover to 2DEG behavior. The behavior of the plasmons is definitely determined by the presence of the Dirac cone $\omega=v_{F} q$, where $v_{F}$ is the Fermi velocity. This indicates that although the dressed electron plasma is massive, it still has Dirac origin. We also investigate graphene double layers subjected to a CPEF with two plasmon branches given as resonances of the real part of the inverse dielectric function. Two cases are investigated: the symmetric configuration when both layers are gapped and the asymmetric case when the gap is induced on one of the layers. The latter case may be obtained by compensating CPEF with the SSB on the given layer. The main derived effect on the acoustic plasmon branch is that this mode shows a gain for the symmetric case, but a loss for the asymmetric case. This applies for a sufficiently intense laser field, over a restricted range of frequency and wavelength. 19 In Sec. IV, we offer some remarks concerning experimental observation of the plasma excitations.

\section{Model and Formulation of the Problem}

Let us consider several graphene layers separated by distance $d$. One of the layers may be epitaxially grown on a SiC substrate. The structure is optically pumped by an idealized single mode CPEF characterized by an associated energy $N_{0} \hbar \omega_{0}$ where $N_{0}$ is the average number of photons in the optical field. It is assumed to be a function of the external pump intensity. We also take it that all graphene layers are in the node of the optical field and we can neglect retardation effects. This is valid provided that $d \ll 2 \pi c / \omega_{0}$. The objective is to find the plasmon dispersion branches $\omega_{ \pm}(q)$ for double layer graphene. Those are given by the poles of the inverse dielectric function. A single layer may be described by the same formalism by setting the inter-layer distance to infinity. By setting the origin $z=0$ at one of the layers, we may follow the procedure presented in Ref. 20 to obtain the inverse dielectric function as: 


$$
\varepsilon^{-1}\left(z, z^{\prime} ; q, \omega\right)=\delta\left(z-z^{\prime}\right)+\sum_{j, j^{\prime}=0,1} \delta(z-(j-1) d) v_{c}\left(j d, j^{\prime} d\right) \Pi_{j j^{\prime}}^{(0)}(q, \omega)
$$

where $\delta\left(z-z^{\prime}\right)$ is the Dirac delta function and polarization matrix is

$$
\left(\begin{array}{cc}
\Pi_{11} & \Pi_{12} \\
\Pi_{21} & \Pi_{22}
\end{array}\right)=\frac{1}{\varepsilon(q, \omega)}\left(\begin{array}{cc}
\Pi_{11}^{(0)}\left(1-v_{c}(q) \Pi_{22}^{(0)}\right) & v_{c}(q) e^{-q d} \Pi_{11}^{(0)} \Pi_{22}^{(0)} \\
v_{c}(q) e^{-q d} \Pi_{11}^{(0)} \Pi_{22}^{(0)} & \Pi_{22}^{(0)}\left(1-v_{c}(q) \Pi_{11}^{(0)}\right)
\end{array}\right)
$$

It is expressed in terms of the generalized dielectric function

$$
\varepsilon(q, \omega)=1-v_{c}(q)\left\{\left[\Pi_{11}^{(0)}+\Pi_{22}^{(0)}\right]+\left[\left(1-e^{-2 q d}\right) v_{c}(q) \Pi_{11}^{(0)} \Pi_{22}^{(0)}\right]\right\}
$$

where $v_{c}(q)=2 \pi e^{2} / \varepsilon_{s} q$ with $\varepsilon_{s} \equiv 4 \pi \varepsilon_{0} \varepsilon_{b}, \varepsilon_{b}$ being the average background dielectric constant. The plasmon resonances correspond to the poles of the inverse dielectric function which are given by the solutions of $\varepsilon(q, \omega)=0$. Additionally, the plasmon frequency incorporates its a relaxation rate so that $\omega \rightarrow \omega+i \eta$. Also, $\Pi_{j j}^{(0)}(q, \omega+i \eta)$ is the noninteracting polarization function for the $j^{\text {th }}$ graphene layer. The inter-layer polarization function $\Pi_{12}^{(0)}$ is not included in our formalism due to negligible interlayer tunneling.

The key ingredient of the above equations is the polarization function. This requires specifics from the model given by the Hamiltonian at the two inequivalent $\mathbf{K}, \mathbf{K}^{\prime}$ points in the graphene dispersion:

$$
\begin{gathered}
H=\mathscr{H}_{J C}+\mathscr{H}_{D} \\
\mathscr{H}_{J C}=\hbar \omega_{0} a^{\dagger} a+\frac{\Delta \mu_{A, B}}{2} \sigma_{3}-\frac{W_{0}}{2 \sqrt{N_{0}}}\left(\sigma_{+} a+\sigma_{-} a^{\dagger}\right) \\
\mathscr{H}_{D}=\hbar v_{F}\left(\left(\sigma_{+}+\sigma_{-}\right) k_{x} \pm i\left(\sigma_{-}-\sigma_{+}\right) k_{y}\right) .
\end{gathered}
$$

Here, the Jaynes-Cummings Hamiltonian (??) is described by the interaction third term governed by the amplitude of the electric filed $E_{0}=\sqrt{N_{0} \hbar \omega_{0} / \varepsilon_{0} V}$, and $V$ is the quantization volume of the CPEF. The energy of an electron in rotational motion induced by the field is denoted as $W_{0}=$ 
$2 v_{F} e E_{0} / \omega_{0} \sim \sqrt{N_{0}}$ and assumed to be much smaller than the energy of the optical filed itself, i.e., $W_{0} / \hbar \omega_{0} \ll 1$. The second term in ?? takes account of the breaking of the inversion symmetry between $A$ and $B$ sub-lattices. Also, $\Delta \mu_{A, B}$ stands for the difference in on-site energies for the sub-lattices. It may be induced if the graphene is grown epitaxially on a substrate, and may acquire values as large as $260 \mathrm{meV}(4775 \mathrm{~nm})$, see, for instance, Zhou et al. ${ }^{15}$ and references therein. On the smaller scale $\left(\sim 10^{-1} \mathrm{meV}\right)$ the symmetry breaking term accounts for spin-orbit interaction.

The Dirac part of the Hamiltonian is given by ??, with \pm standing for $\mathbf{K}, \mathbf{K}^{\prime}$ points correspondingly. The operators involved in the above Hamiltonian are acting in the joined electro/photon space via the following relations:

$$
\begin{array}{cc}
\sigma_{+}=|\uparrow\rangle\langle\downarrow| ; & \sigma_{-}=|\downarrow\rangle\langle\uparrow| ; \\
\sigma_{+}\left|\uparrow, N_{0}\right\rangle=0 ; & \sigma_{+}\left|\downarrow, N_{0}\right\rangle=\left|\uparrow, N_{0}\right\rangle \\
\sigma_{-}\left|\downarrow, N_{0}\right\rangle=0 ; & \sigma_{-}\left|\uparrow, N_{0}\right\rangle=\left|\downarrow, N_{0}\right\rangle \\
a\left|\downarrow \uparrow, N_{0}\right\rangle=\sqrt{N_{0}}\left|\downarrow \uparrow, N_{0}-1\right\rangle ; & a^{\dagger}\left|\downarrow \uparrow, N_{0}\right\rangle=\sqrt{N_{0}+1}\left|\downarrow \uparrow, N_{0}+1\right\rangle
\end{array}
$$

with $|\uparrow \downarrow\rangle$ denoting the Dirac pseudo-spin basis. The Jaynes-Cummings part can be readily diagonalized by switching to the dressed electron basis

$$
\begin{gathered}
\left(\begin{array}{c}
\left|+, N_{0}\right\rangle \\
\left|-, N_{0}\right\rangle
\end{array}\right)=\left(\begin{array}{cc}
\cos \left(\Phi_{N_{0} / 2}\right) & \sin \left(\Phi_{N_{0}} / 2\right) \\
-\sin \left(\Phi_{N_{0}} / 2\right) & \cos \left(\Phi_{N_{0}} / 2\right)
\end{array}\right)\left(\begin{array}{c}
\left|\uparrow, N_{0}\right\rangle \\
\left|\downarrow, N_{0}+1\right\rangle
\end{array}\right) \\
\cos \left(\Phi_{N_{0}} / 2\right)=\sqrt{\frac{\Omega_{N_{0}}+\Delta}{2 \Omega_{N_{0}}}} \\
\sin \left(\Phi_{N_{0}} / 2\right)=\sqrt{\frac{\Omega_{N_{0}}-\Delta}{2 \Omega_{N_{0}}}}
\end{gathered}
$$

where $\Omega_{N_{0}}^{2}=\Delta^{2}+4 W_{0}^{2}\left(N_{0}+1\right) / N_{0}$ and the detuning between the microcavity and the symmetry 
breaking induced gap is denoted by $\Delta=\hbar \omega-\Delta \mu_{A, B}$. In the new basis, the Hamiltonian ?? within the small dressing regime $W_{0} \ll \Delta$ assumes the following form

$$
H=\mathbb{I} N_{0} \hbar \omega_{0}+\frac{E_{g}}{2} \sigma_{3}+\mathscr{H}_{D}
$$

where $E_{g}=\sqrt{W_{0}^{2}+\Delta^{2}}-\hbar \omega_{0}$ is the energy gap between valence and conduction bands. The gap governs the nature of dressed Dirac electrons metal-insulator transition. 1 One may obtain the solution of the above equation in a straightforward way, giving

$$
\begin{aligned}
& E_{ \pm, \mathbf{k}}=N_{0} \hbar \omega_{0} \pm \sqrt{\left(E_{g} / 2\right)^{2}+\left(\hbar v_{F} k\right)^{2}} \\
& \left(\begin{array}{c}
|+, \mathbf{k}\rangle \\
|-, \mathbf{k}\rangle
\end{array}\right)=\frac{e^{i \mathbf{k r}}}{\sqrt{1+\alpha_{N_{0}, k}^{2}}}\left(\begin{array}{cc}
1 & \mathscr{A}_{N_{0}, k} e^{i \theta_{\mathbf{k}}} \\
\mathscr{A}_{N_{0}, k} & -e^{i \theta_{\mathbf{k}}}
\end{array}\right)\left(\begin{array}{c}
\left|+\frac{1}{2}, N_{0}\right\rangle \\
\left|-\frac{1}{2}, N_{0}\right\rangle
\end{array}\right) \\
& \mathscr{A}_{N_{0}, k}=\frac{\hbar v_{F} k}{\sqrt{\left(\hbar v_{F} k\right)^{2}+\left(E_{g} / 2\right)^{2}}+E_{g} / 2}
\end{aligned}
$$

It is now a simple matter to show that conventional Dirac fermions correspond to the offresonance limit of the dressed states. Given the dressed states, the noninteracting polarization assumes the Lindhard form

$$
\begin{aligned}
& \Pi_{j j}^{(0)}(q, \omega)=\Pi_{j j}^{(0), 1}(q, \omega)+\Pi_{j j}^{(0), 2}(q, \omega) \\
& \Pi_{j j}^{(0), 1}(q, \omega)=-\frac{1}{\pi} \sum_{\alpha= \pm} \int_{0}^{\infty} k d k \mathscr{I}^{\alpha-}(k, q, \omega) ; \quad \Pi_{j j}^{(0), 2}(q, \omega)=\frac{1}{\pi} \sum_{\alpha, \beta= \pm} \Re \int_{0}^{\sqrt{\mu^{2}-\left(E_{g} / 2\right)^{2}} / \hbar v_{F}} k d k \mathscr{I}^{\alpha \beta}(k, q, \omega) \\
& \mathscr{I}^{\alpha \beta}(k, q, \omega)=\int_{0}^{2 \pi} d \phi \frac{\mathscr{F}_{\mathbf{k}, \mathbf{k}+\mathbf{q}}^{\beta}}{\hbar \omega+\alpha E_{\mathbf{k}}-\alpha \beta E_{\mathbf{k}+\mathbf{q}}} ; \quad \quad \mathscr{F}_{\mathbf{k}, \mathbf{k}+\mathbf{q}}^{\beta}=\frac{1}{2}\left(1+\beta \frac{\hbar^{2} v_{F}^{2} \mathbf{k} \cdot(\mathbf{k}+\mathbf{q})+\left(E_{g} / 2\right)^{2}}{E_{\mathbf{k}} E_{\mathbf{k}+\mathbf{q}}}\right)
\end{aligned}
$$

The two terms in ?? stand for the inter- and intra-band polarizations, respectively. Also, $\mu$ is the chemical potential whose zero value is in the middle of the gap $\left(E_{g}\right)$. In calculating the polarization, we assumed that for chosen $N_{0}$, each point on the dispersion curve $E_{\mathbf{k}}$ might be occupied 
by no more than one electron. This assumption is valid for small energy gap. Otherwise, the quasi-equilibrium density matrix acquires non vanishing off-diagonal elements thus substantially complicating the statistical properties of the dressed states. The noninteracting polarization ?? in gaped graphene has already been calculated on both the real and imaginary frequency axes 321 which essentially yield the same result. Since it plays a crucial role in this paper, we give its full form along the real frequency axis as

$$
\begin{gathered}
\Pi_{j}^{(0)}(q, \omega)=-\frac{2 \mu}{\pi \hbar^{2} v_{F}^{2}}+\frac{q^{2}}{4 \pi \sqrt{\left|\hbar^{2} v_{F}^{2} q^{2}-\hbar^{2} \omega^{2}\right|}} \times[ \\
\left.\left.\left(i G_{>}\left(x_{1,-}\right)-i G_{>}\left(x_{1,+}\right)\right)\right) 1_{<}+\left(G_{<}\left(x_{1,-}\right)+i G_{>}\left(x_{1,+}\right)\right)\right) 2_{<}+ \\
\left.\left.\left.\left(G_{<}\left(x_{1,+}\right)+G_{<}\left(x_{1,-}\right)\right)\right) 3_{<}+\left(G_{<}\left(x_{1,-}\right)-G_{<}\left(x_{1,+}\right)\right)\right) 4_{<}+\left(G_{>}\left(x_{1,+}\right)-G_{>}\left(x_{1,-}\right)\right)\right) 1_{>}+ \\
\left.\left.\left(G_{>}\left(x_{1,+}\right)+i G_{<}\left(x_{1,-}\right)\right)\right) 2_{>}+\left(G_{>}\left(x_{1,+}\right)-G_{>}\left(-x_{1,-}\right)-i \pi\left(2-x_{0}^{2}\right)\right)\right) 3_{>}+ \\
\left.\left.\left.\left(G_{>}\left(-x_{1,-}\right)+G_{>}\left(x_{1,+}\right)-i \pi\left(2-x_{0}^{2}\right)\right)\right) 4_{>}+\left(G_{0}\left(x_{1,+}\right)-G_{0}\left(x_{1,-}\right)\right)\right) 5_{>}\right]
\end{gathered}
$$

Here, the following notations for the region functions have been introduced:

$$
\begin{aligned}
x_{0} & =\sqrt{1+\frac{E_{g}^{2}}{\hbar^{2} v_{F}^{2} q^{2}-\hbar^{2} \omega^{2}}}, \\
x_{1, \pm} & =\frac{2 \mu \pm \hbar \omega}{\hbar v_{F} q}, \\
x_{2, \pm} & =\sqrt{\hbar^{2} v_{F}^{2}\left(q \pm k_{F}\right)^{2}+\left(E_{g} / 2\right)^{2}} \\
x_{3} & =\sqrt{\hbar^{2} v_{F}^{2} q^{2}+E_{g}^{2}}, \\
G_{<}(x) & =x \sqrt{x_{0}^{2}-x^{2}}-\left(2-x_{0}^{2}\right) \cos ^{-1}\left(x / x_{0}\right), \\
G_{>}(x) & =x \sqrt{x^{2}-x_{0}^{2}}-\left(2-x_{0}^{2}\right) \cosh ^{-1}\left(x / x_{0}\right), \\
G_{0}(x) & =x \sqrt{x^{2}-x_{0}^{2}}-\left(2-x_{0}^{2}\right) \sinh ^{-1}\left(x / \sqrt{-x_{0}^{2}}\right),
\end{aligned}
$$


and the regions are shown in Fig. [figure][2][]2 and are defined as

$$
\begin{array}{lc}
1_{<}= & \theta\left(\mu-x_{2,-}-\hbar \omega\right) \\
2_{<}= & \theta\left(-\hbar \omega-\mu+x_{2,-}\right) \theta\left(\hbar \omega+\mu-x_{2,-}\right) \theta\left(\mu+x_{2,+}-\hbar \omega\right) \\
3_{<}= & \theta\left(-\mu+x_{2,-}-\hbar \omega\right) \\
4_{<}= & \theta\left(\hbar \omega+\mu-x_{2,+}\right) \theta\left(\hbar v_{F} q-\hbar \omega\right) \\
1_{>}= & \theta\left(2 k_{F}-q\right) \theta\left(\hbar \omega-x_{3}\right) \theta\left(\mu+x_{2,-}-\hbar \omega\right) \\
2_{>}= & \theta\left(\hbar \omega-\mu-x_{2,-}\right) \theta\left(\mu+x_{2,+}-\hbar \omega\right) \\
3_{>}= & \theta\left(\hbar \omega-\mu-x_{2,+}\right) \\
4_{>}= & \theta\left(q-2 k_{F}\right) \theta\left(\hbar \omega-x_{3}\right) \theta\left(\mu+x_{2,-}-\hbar \omega\right) \\
5_{>}= & \theta\left(\hbar \omega-\hbar v_{F} q\right) \theta\left(x_{3}-\hbar \omega\right)
\end{array}
$$

For the purposes of optical spectroscopy, it is advisable to study the long wavelength limit by expanding the polarization function in ?? in powers of $q$. For small $q / k_{F}$, the undamped plasmons exist in the spectral regions $1_{>}$and $5_{>}$. By expanding the polarization up to the fourth order in $q$, we obtain

$$
\Pi_{j j}^{(0)}(q, \omega)=\frac{q^{2}}{4 \hbar^{3} \pi \omega^{3}} \times\left\{\begin{array}{cc}
4 \hbar \mu \omega+\left(E_{g}^{2}+\hbar^{2} \omega^{2}\right) \log \left[\frac{2 \mu-\hbar \omega}{2 \mu+\hbar \omega}\right]+O\left(\frac{q^{4}}{\omega^{4}}\right) ; & 1_{>}, 5_{>}, 4_{<} ; \\
-\frac{16 \hbar \mu \omega^{3}}{v_{F}^{2} q^{2}}-4 \hbar \mu \omega-\left(E_{g}^{2}+\hbar^{2} \omega^{2}\right) \log \left[\frac{2 \mu-\hbar \omega}{2 \mu+\hbar \omega}\right]+O\left(\frac{q^{4}}{\omega^{4}}\right) ; & 1_{<,}, 2_{<} .
\end{array}\right.
$$

For small frequency $\omega$, the following identity applies, $\left(E_{g}^{2}+\hbar^{2} \omega^{2}\right) \log \left[\frac{\mp 2 \mu \pm \hbar \omega}{2 \mu+\hbar \omega}\right] \approx-\hbar E_{g}^{2} \omega / \mu$, which we employed to further simplify the polarization (??) as 


$$
\begin{gathered}
\Pi_{j j}^{(0)}(q, \omega)=\frac{q^{2} \mu}{\pi \hbar^{2} \omega^{2}}\left(1-\frac{E_{g}^{2}}{4 \mu^{2}}\right)\left(1_{>}+5_{>}+4_{<}\right)- \\
-\left(\frac{4 \mu}{\pi \hbar^{2} v_{F}^{2}}-\frac{q^{2} \mu}{\pi \hbar^{2} \omega^{2}}\left(1-\frac{E_{g}^{2}}{4 \mu^{2}}\right)\right)\left(1_{<}+2_{<}\right)
\end{gathered}
$$

Note that smallness of $\omega$ is automatically assured in the regions $4_{<,}, 1_{<,} 2_{<}$. In these regions, the dominant excitations are of the particle-hole type, due to nonzero imaginary part of the polarization. In the long wavelength limit, the plasmon frequency for a single graphene layer in the vacuum is obtained by solving for the zeros of $\varepsilon(q, \omega)$ in ??, along with $\Pi_{11}^{(0)}=\Pi^{(0)}(q, \omega)$ given by ?? and $\Pi_{22}^{(0)}$ set to zero, yielding

$$
\omega_{p}^{2}=q \mathscr{P}\left(E_{g}, \omega_{p}\right)
$$

where we introduced the plasmon region function

$$
\mathscr{P}\left(E_{g}, \omega_{p}\right)=\frac{2 \mu e^{2}}{\hbar^{2} \varepsilon_{0}}\left(1-\frac{E_{g}^{2}}{4 \mu^{2}}\right)\left(\left.\left(1_{>}+5_{>}+4{ }_{<}\right)\right|_{\omega \rightarrow \omega_{p}}+\left.\frac{q}{q+80 \pi \mu / \hbar v_{F}}\left(1_{<}+2_{<}\right)\right|_{\omega \rightarrow \omega_{p}}\right) .
$$

In the above expression we used $e^{2} / 4 p i \varepsilon_{0} \hbar v_{F}=2.5$ It is also straightforward to show that the region function disappears in the region $1_{<}$, thus supporting Landau damped plasmons in the region $2<$ only. We also note that for conventional graphene and the 2DEG, the plasmon region function ?? is replaced by constants $2 \mu e^{2} / \hbar^{2} \varepsilon_{S}$ and $m^{\star} v_{F}^{2} e^{2} / \hbar^{2} \varepsilon_{s}$, respectively.

For the symmetric double layer configuration, one obtains two undamped and two Landau damped plasmon branches whose frequencies in the long wavelength limit obey

$$
\omega_{ \pm}^{2}=q e^{-q d}\left(e^{q d} \pm 1\right) \mathscr{P}\left(E_{g}, \omega_{ \pm}\right)
$$


where \pm stands for the bonding/anti-bonding plasmon modes, when the polarizations in the two layers work together/against each other in producing the charge density oscillations. Two independent parameters are $q d$ and $E_{g}$. That is the interplay between the inter-layer distance and the plasmon region function determines the plasmon branches. In the limit of large inter-layer separation, $q d \gg 1$, both branches are reduced to ??. Conventionally, $\underline{22}$ those regimes are referred to as intermediate and weak coupling limits. We avoid using these terms so as not to confuse with the light-matter coupling. On the other hand, for small inter-layer separations, $q d \ll 1$, we have

$$
\begin{gathered}
\omega_{+}^{2}=q \mathscr{P}\left(E_{g}, \omega_{ \pm}\right)(2-q d) \\
\omega_{-}^{2}=q^{2} \mathscr{P}\left(E_{g}, \omega_{ \pm}\right) d
\end{gathered}
$$

Due to linear dependence of the undamped plasmon frequency on the wave vector $q$, the antibonding mode is often referred to as acoustical branch. The other undamped plasmon branch has larger frequency and is named the optical branch. To the leading order in $q$, this branch is independent of inter-layer separation and is completely determined by the plasmon region function. It is always higher in frequency than the acoustical branch and usually (but not always) experiences Landau damping in the long wavelength limit.

The asymmetric case is also easily obtained in the long wavelength limit, i.e.,

$$
\omega_{ \pm}^{2}=\frac{1}{2} q e^{-q d}\left(e^{q d}\left(\mathscr{P}_{1, \pm}+\mathscr{P}_{2, \pm}\right) \pm \sqrt{e^{2 q d}\left(\mathscr{P}_{1, \pm}-\mathscr{P}_{2, \pm}\right)^{2}+4 \mathscr{P}_{1, \pm} \mathscr{P}_{2, \pm}}\right)
$$

where we have introduced the short hand notation $\mathscr{P}_{1(2), \pm}=\mathscr{P}\left(E_{g, 1(2)}, \omega_{ \pm}\right)$, with $E_{g, 1(2)}$ denoting the gap induced on the first (second) graphene layer. In the small inter-layer separation limit, these eigenfrequencies become 


$$
\begin{gathered}
\omega_{+}^{2}=q\left(\mathscr{P}_{1,+}^{2}+\mathscr{P}_{2,+}^{2}-2 \mathscr{P}_{1,+} \mathscr{P}_{2,+}(q d-1)\right)\left(\mathscr{P}_{1,+}+\mathscr{P}_{2,+}\right)^{-1} \\
\omega_{-}^{2}=2 d q^{2} \mathscr{P}_{1,-} \mathscr{P}_{2,-}\left(\mathscr{P}_{1,-}+\mathscr{P}_{2,-}\right)^{-1}
\end{gathered}
$$

When one moves away from the long wavelength limit, $\varepsilon(q, \omega)=0$ from ?? in conjunction with (??) become nonlinear, with no closed-form analytic solutions. Consequently, numerical simulations are needed. Our numerical results are the subject of the next section.

\section{Numerical results and Discussion}

In our numerical calculations, we scaled energies in units of the chemical potential $\mu$ and the wave number in units of the Fermi wave number $k_{F}$. The frequency of the electromagnetic field along with its intensity determines the energy gap $E_{g}$ between the valence and conduction bands. Consequently, this is the only parameter we use to specify the role played by the external laser pump field. All our calculations for the collective plasma excitations were carried out at $T=0 \mathrm{~K}$.

We begin this section by considering the plasmon dispersion for conventional Dirac electrons in graphene obtained by solving $\mathfrak{R} e \varepsilon(q, \omega)=0$ with the use of ??. Our results are presented in $\left[\right.$ figure][3][]3 (a.1) and are superimposed onto the particle-hole modes by plotting $\mathfrak{I} m \Pi^{(0)}(q, \omega)$.

This is done in order to identify the regions where there is Landau damping. Clearly, the zeros of ?? yield both damped and undamped plasmon excitations. In order to isolate the undamped modes, it is convenient to consider the plasmon spectral weight $-(1 / \pi) \mathfrak{I} m \Pi\left(q, \omega-i \gamma_{q}\right)$, where $\Pi(q, \omega-$ $\left.i \gamma_{q}\right)=\Pi^{(0)}\left(q, \omega-i \gamma_{q}\right) / \varepsilon\left(q, \omega-i \gamma_{q}\right)$. The plasmon relaxation rate is determined self-consistently, starting with the phenomenological rate whose value hereafter is chosen as $\hbar \gamma / \mu=10^{-4}$. If not for the finite relaxation rate, the plasmon spectral weight is nonzero only in the particle-hole regions . 6 Below, we show that this condition applies only for the single layer configuration. The concept is demonstrated in [figure][3][]3(b.1). The naturally damped plasmon branch in the $1_{>}$triangular region becomes strongly Landau damped once it enters the particle-hole mode region $2_{>}$. 
By dressing Dirac electrons with circularly polarized photons, they acquire an effective mass and a gap $E_{g}$ is opened between the valence and conduction bands. The gap is determined by the intensity and frequency of the laser field. The presence of $E_{g}$ in turn induces a gap in the particle-hole mode region for $q / k_{F} \gtrsim 1$. The particle-hole free region $5>$ widens with increasing $E_{g}$. Its contribution to the plasmon region function also grows, as indicated by the second term in ??. Meanwhile, the Dirac contribution of the plasmon region function is decreased through the first term in the same equation. Schematically, the opening of the plasmon window by the electron dressing is shown in [figure][2][]2, There is one more region in the particle-hole continuum which gets opened by the energy gap $E_{g}$, namely region $4_{<}$. We give it special attention since this region provides the main difference between dressed Dirac electrons and conventional graphene. In the latter case, this region simply merges with $5_{>}$, since the $\omega=\hbar v_{F} q$ line has no physical meaning. However, in gapped graphene, this region is very special due to the possible natural plasmon gain existing in it. Indeed, according to ??, the polarization acquires an imaginary part provided analytical continuation $\omega \rightarrow \omega-i \gamma$ from the real axis into the lower complex half-plane $\gamma>0$. The imaginary part of the polarization becomes a weighted delta function, centered at the plasma frequency $\mathfrak{I} m \Pi(q, \omega)=\mathfrak{I} m\left(1 /\left(v_{c}(q) \varepsilon\left(q, \omega_{p}\right)\right)\right)=\operatorname{sgn}\left(\mathfrak{I} m \varepsilon\left(q, \omega_{p}\right)\right)\left(\pi / v_{c}(q)\right) \delta\left(\mathfrak{R} e \varepsilon\left(q, \omega_{p}\right)\right)=$ $\pi W\left(q, \omega_{p}\right) \delta\left(\omega-\omega_{p}\right)$.

In the region $5_{>}$the plasmon weight is the conventional positive one given by $W\left(q, \omega_{p}\right)=W_{0} 5_{>}$ , with $\left.W_{0}=1 /\left.v_{c}^{2}(q) \frac{\partial}{\partial \omega} \Re e \Pi^{(0)}(q, \omega)\right|_{\omega=\omega_{p}}\right)>0$. This behaviour can be seen on the lower insets of [figure][3][]3 (a.3). However, when the plasmon branch enters the region $4>$ the plasmon weight may flip its sign $W\left(q, \omega_{p}\right)=-W_{0} 4_{>}$, since $\mathfrak{I} \Pi^{(0)}\left(q, \omega_{p}\right)<0$ as indicated on the upper inset of [figure][3][]3(a.3). Interestingly enough the change in sign of the plasmon weight requires the plasmon branch to cross $\omega=\hbar v_{F} q$ line. On one hand, the gapeless graphene does not show the crossover from the plasmon loss to the gain since the above condition is never satisfied. On the other hand, if one is to increase energy gap $\left(E_{g} / \mu \approx 2\right)$ so that the plasmon branch is completely forced into the $4_{<}$region, then the plasmons also show only the loss and the positive plasmon weight $W\left(q, \omega_{p}\right)=W_{0} 4_{>}$. For even larger energy gap, the plasmons are forced into region $2_{<}$and 
become fully Landau damped. The plasmon dispersion for a single layer of gapped graphene is presented in [figure][3][]3 (a.2), (a.3). Numerical results shown in [figure][3][]3 (b.2), (b.3) demonstrate the predominant plasmon gain for short wavelengths. In the long wave length limit the plasmon branches are always well behaved. Therefore, at this level of theory, it is impossible to say if the anomalous plasmon behaviors has some physical meaning or being just an indicator of RPA failure at larger $q$.

Turning now to the double layer configuration, we present in [figure][4][]4 through [figure][7][]7 the plasmon dispersion and damping for the symmetric and asymmetric cases. In these plots, the panels (a.1, a.2, a.3, a.4) correspond to zero energy gap for chosen inter-layer separation. In the presence of an energy gap, we note that one of the plasmon modes (the acoustic branch $\omega_{-}$) may be forced into region $4_{<}$for values of $E_{g} / \mu$ as small as one (See ??(b.1, c.1)). As far as the symmetric configuration is concerned, this mode demonstrates gain, while the asymmetric case shows losses in that region as may be deduced from its spectral weight in ?? (b.1, c.1).

For the symmetric double layer, there are two Landau damped plasmon modes in the region $2_{<}$. One of them runs just along $2_{<}, 4_{<}$interface. While the other starts along $2_{<}, 1_{<}$interface and then eventually merges with the first branch at short wavelength, as demonstrated in [figure][4][]4. The frequencies of these plasmon branches are almost independent of the inter-layer separation $d$. The asymmetric configuration in effect causes one of these damped modes to become purely acoustic with frequency $\omega \sim q v_{F}$ over a wide range of wave vector $q$, as shown in [figure][5][]5.

Let us now confine our attention to the plasmon branches whose frequencies in the long wavelength limit coincide with those of the bonding $\left(\omega_{+}\right)$and antibonding $\left(\omega_{-}\right)$modes. For large inter-layer distance satisfying $k_{F} d \gg 1$, in the absence of an energy gap, $E_{g}=0$, these modes converge onto the line $\omega+v_{F} q=2 \mu / \hbar$. By increasing the gap for the symmetric double layer, there emerge Landau damped plasmon branches in regions $2_{>}, 4_{>}$from the bonding mode, as shown in [figure][4][]4(b,c). The remaining part of the $\omega_{+}$branch is almost independent of $d$. This part of the excitation branch mimics the single layer plasmon subjected to $E_{g}$ (symmetric configuration, [figure][4][]4(b.4, c.4)) or gap independent (asymmetric case, [figure][5][]5 (b.4,c.4)). The latter 
case is of special interest since it obeys the conventional plasmon dispersion law for graphene while seemingly progressing into the gap within the particle-hole mode region. However, its spectral weight as seen in [figure][7][]7]demonstrates strong Landau-like damping once it enters region $\omega+v_{F} q>2 \mu / \hbar$.

By bringing the layers close to each other, $\omega_{-}$becomes acoustic like (see ??), and ,for small inter-layer distance, may be mostly accommodated by region $4_{<}$. The $\omega_{+}$mode is similar to that for the single layer. That is, $\omega_{+} \approx \omega_{p}\left(E_{g}\right)$ for the symmetrical case ??, and is gap independent in the asymmetrical case with $\omega_{+} \approx \omega_{p}\left(E_{g}=0\right)$.

\section{Concluding Remarks}

Theoretical results reported here were carefully presented to stimulate experimental verification for both the single and double layer configuration. One possible probe which may be employed is EELS using an electron spectrometer ${ }^{23}$ In EELS, some of the electrons undergo inelastic scattering, losing energy and having their paths slightly modified. The inelastic interactions include phonon excitations, inter band and intra band particle-hole excitations, plasmon excitations and

Cerenkov radiation. 19 A separate study is needed to calculate the stopping power due to these mechanisms in order to supplement the results reported in this paper.

\section{Acknowledgement}

This research was supported by contract \# FA 9453-07-C-0207 of AFRL. DH would like to thank the Air Force Office of Scientific Research (AFOSR) for its support. We also appreciate useful discussions with P. Pytkovskiy. 


\section{References}

1. O. V. Kibis, Phys. Rev. B 81, 165433 (2010).

2. X.-F. Wang and T. Chakraborty, Phys. Rev. B. 75, 033408 (2007).

3. P. K. Pyatkovskiy, J. Phys.: Condens. Matter 21, 025506 (2009).

4. G. Li, A. Luican, and E.Y. Andrei, Phys. Rev. Lett. 102, 176804 (2009).

5. G. Giovannetti, et. al., Phys. Rev. B. 76, 073103 (2007).

6. B. Wunsch, T. Stauber, F. Sols, and F. Guinea, New J. Phys. 8, 318 (2006).

7. K. W-K. Shung, Phys. Rev. B. 34, 979 (1986).

8. K. W-K. Shung, Phys. Rev. B. 34, 1264 (1986).

9. J.-Y. Wu, S.-C. Chen, O. Roslyak, G. Gumbs, and M.-F. Lin, ACS Nano (Web), DOI: 10.1021/nn1024847 (2011).

10. G. Gumbs, Phys. Rev. B. 70, 235314 (2004).

11. M. S. Kushwaha, Phys. Rev. B. 76, 245315 (2007).

12. G. Gumbs, Phys. Rev. B. 73, 165315 (2006).

13. E. Rotenberg, et. al., Nature Matter. 7, 258 (2008).

14. S. Kim, J. Ihm, H. J. Choi, and Y. Son, Phys. Rev. Lett. 100, 176802 (2008).

15. S. Y. Zhou et al., Nature Matter. 6, 770 (2007).

16. A. Bostwick et al., Nature Phys. 3, 36 (2007).

17. B. N. J. Persson, Solid State Commun. 52, 811 (1984).

18. G. Gumbs, Phys. Rev. B. 37, 10184 (1988). 
19. A. Balassis and G. Gumbs, J. Appl. Phys. 106, 103102 (2009).

20. G. Gumbs, Solid State Commun. 65, 393 (1988).

21. A. Quaimzadeh and R. Asgari, New J. Phys. 11, 095023 (2009).

22. S. Das Sarma and A. Madhukar, Phys. Rev. B. 23, 805 (1981).

23. H. Ibach and D. L. Mills, Electron Energy loss spectroscopy and surface vibrations, Academic Press, New York, (1982). 


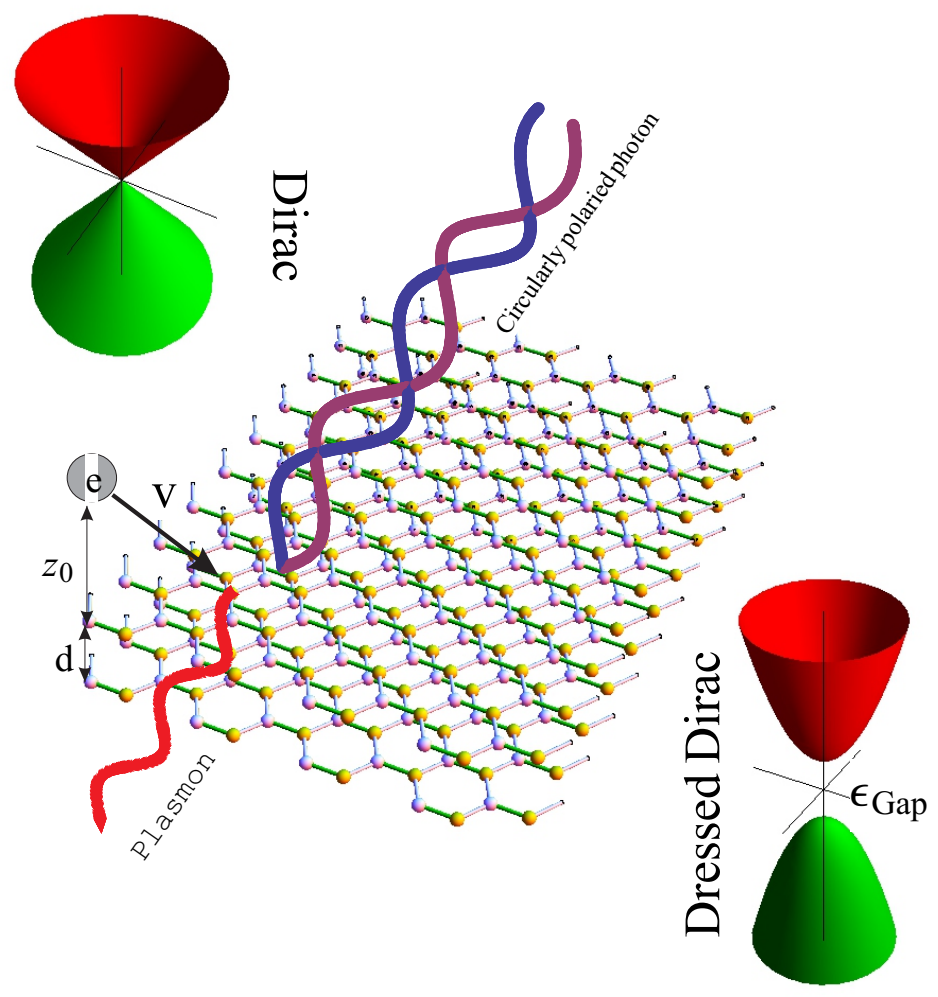

Figure 1: (Color online) Schematic illustration of the pump-probe consisting of circularly polarized light and a beam of energetic charged particles. On the left is the energy band structure for conventional Dirac fermions near the K-point. On the right is the corresponding band structure for dressed Dirac fermions. 

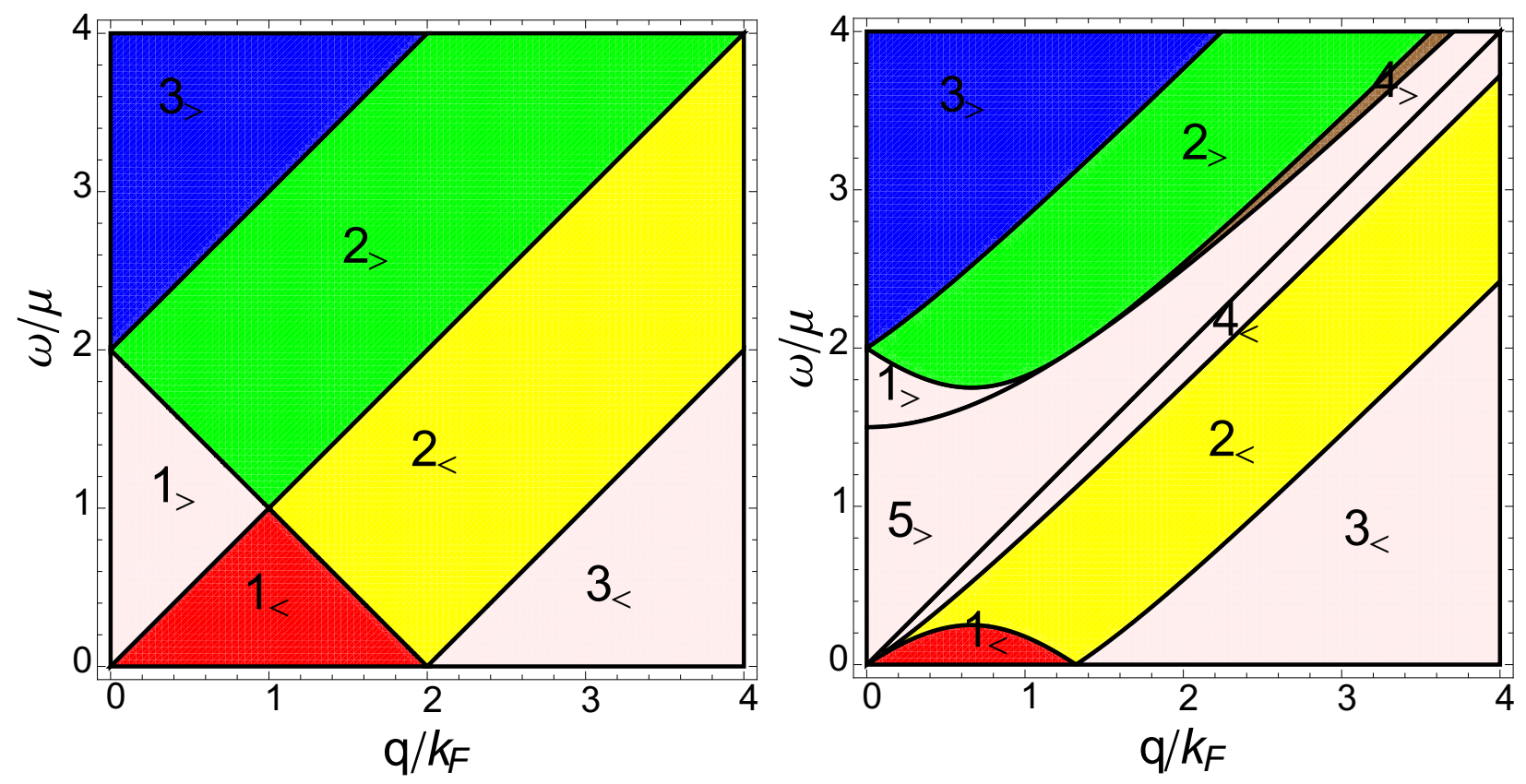

Figure 2: (Color online) Labeled regions for analytic results for the polarization function in the case of conventional graphene (zero energy gap, left panel) and for the case when there is an induced gap $E_{g} / \mu=1.5$ (right panel). 
(a.1)

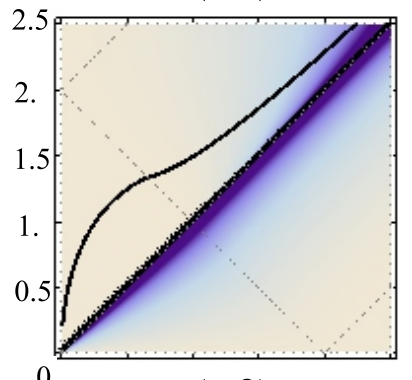

(a.2)

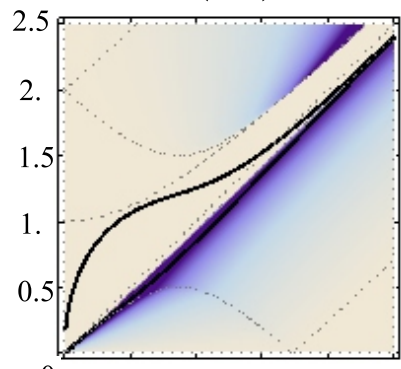

(a.3)

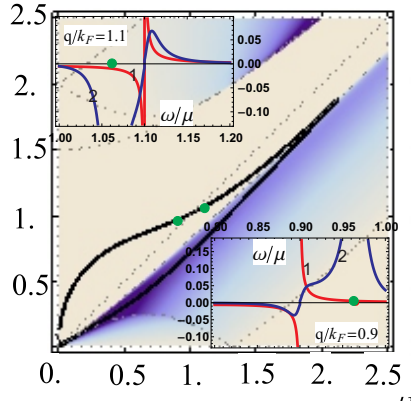

(b.1)

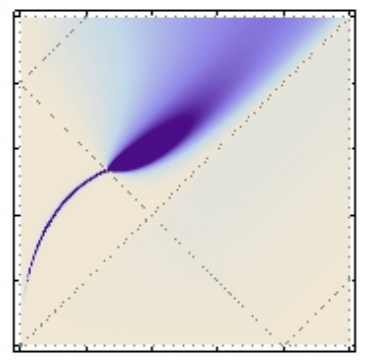

(b.2)

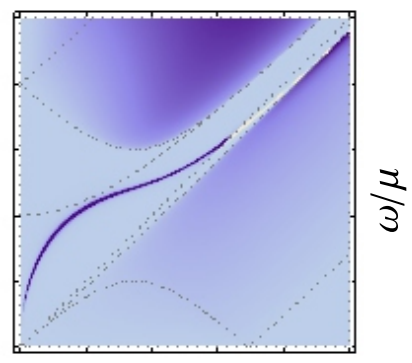

(b.3)

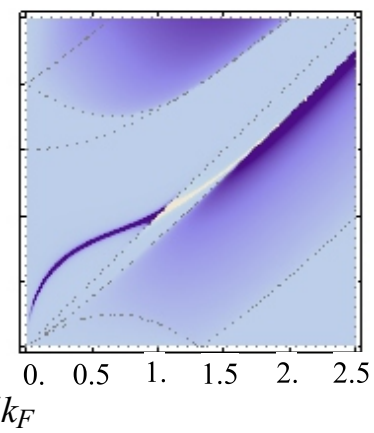

Figure 3: (Color online) The plasma excitations and their damping are presented for a single graphene layer. In rows $(1,2,3)$, the energy gaps chosen are $E_{g} / \mu=\{0.0,1.0,1.5\}$, respectively. In the panels labeled (a), we plot $\mathfrak{I} m \Pi^{(0)}(q, \omega)$ as well as $\mathfrak{R} e \varepsilon(q, \omega)=0$ (with $d \rightarrow \infty$ ). Panels (b) were obtained by plotting the spectral weight $-(1 / \pi) \mathfrak{I} m \Pi\left(q, \omega-i \gamma_{q}\right)$ which involves the imaginary part of the RPA form of the polarization function. In panels (a), the darker the shaded regions, the larger is the value of $\mathfrak{I} m \Pi^{(0)}(q, \omega)$, corresponding to Landau damping. The black solid curves in panels (a) show the plasmon dispersion obtained by solving $\mathfrak{R} e \varepsilon(q, \omega)=0$. The finite lifetime of the plasmon modes represented by the thin solid black curves in panels (b), was included in the polarization function to take account of phonon-like scattering and was determined by solving $\mathfrak{I} m \Pi\left(q, \omega+i \gamma_{q}\right)=0$. The auxiliary dashed lines show $n_{>}$and $n_{<}$regions. The upper and lower insets of (a.3) correspond to the fixed values of $q$ so that the plasmon branch is below and above $\omega=\hbar v_{F} q$ line. The green dot indicates the position of the plasmon resonance $\omega_{p}$. Red (1) curve indicates $\mathfrak{I} \Pi^{(0)}$, while blue (2) curve stands for $\mathfrak{I} \Pi$. 
(a.1)

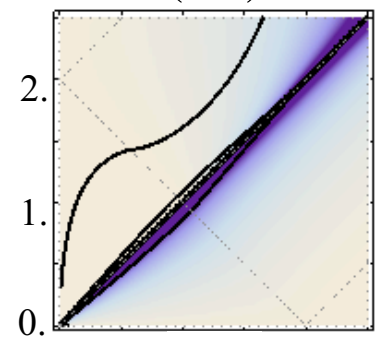

(a.2)

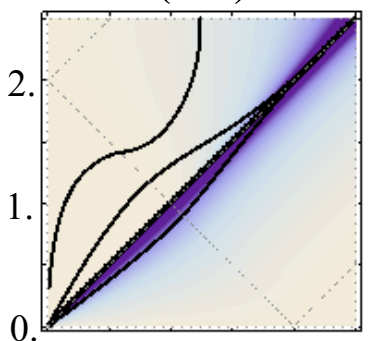

(a.3)

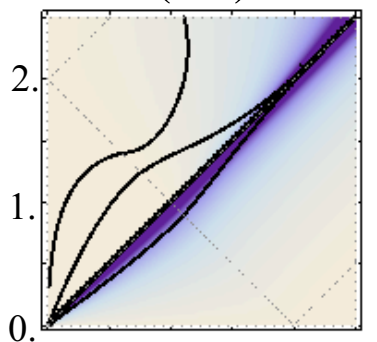

(a.4)

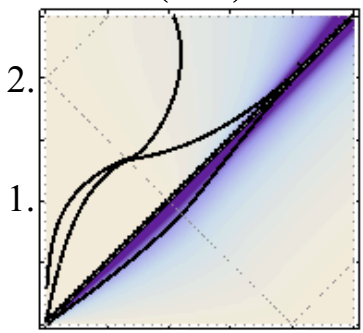

0 . (b.1)

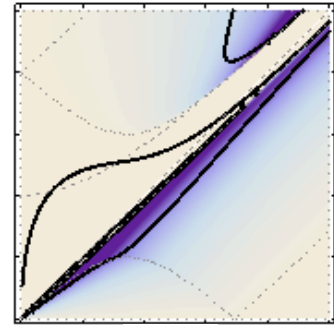

(b.2)

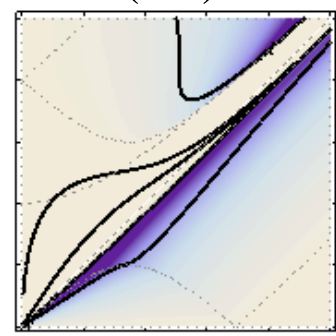

(b.3)

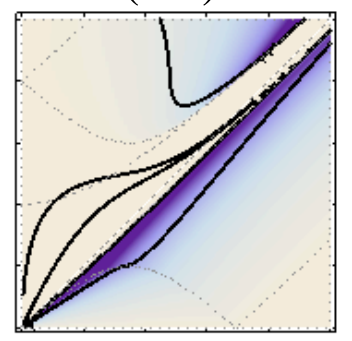

(b.4)

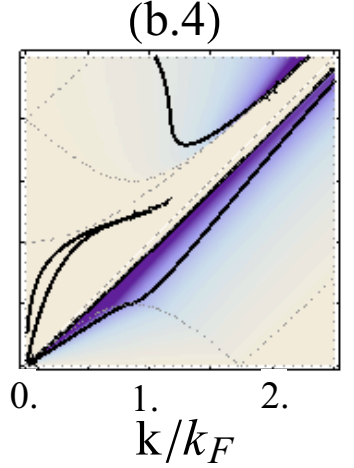

0. (c.1)

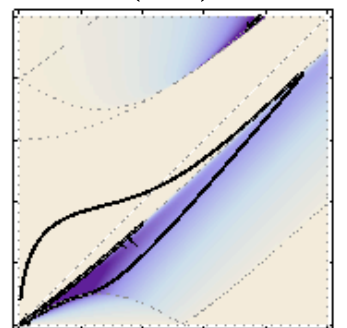

(c.2)

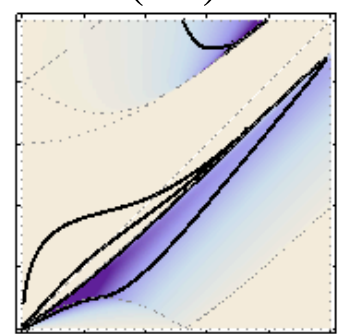

(c.3)

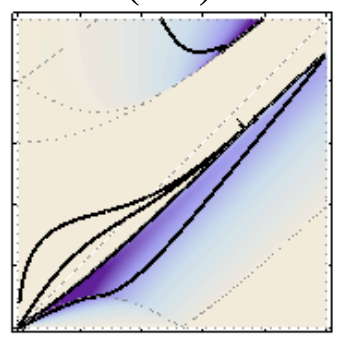

(c.4)

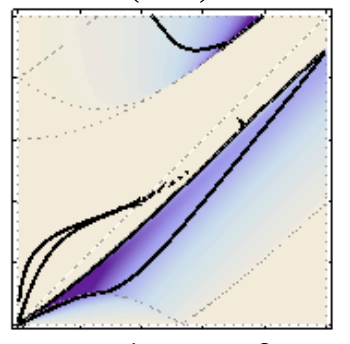

$0 . \quad 1 . \quad 2$.

Figure 4: (Color online) Plasma excitations and damping (analogous to column (a) of [figure][3][]3 for double layer configuration of graphene layers. The imaginary part of the polarization $\Pi^{(0)}$ is plotted as a function of frequency and wave vector. The solid black lines are the plasmon dispersion curves. Columns $(\mathrm{a}, \mathrm{b}, \mathrm{c})$ are for $E_{g} / \mu=\{0.0,1.0,1.5\}$, respectively. The induced gap and chemical potential are the same for both layers (symmetric case). Rows $(1,2,3,4)$ depict plasmon branches for inter-layer distances $k_{F} d=\{0.1,0.5,1.0,5.0\}$, respectively. 
(a.1)

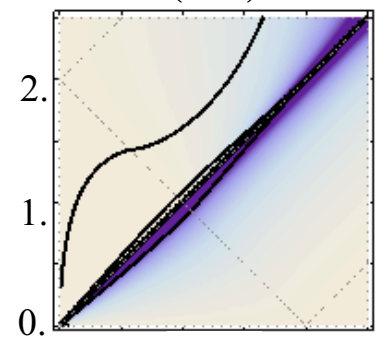

(a.2)

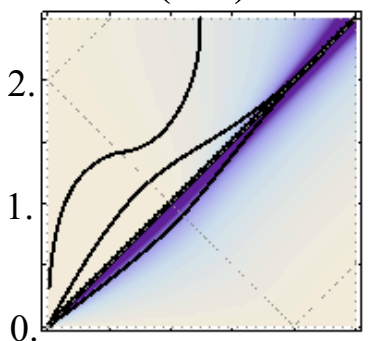

(a.3)

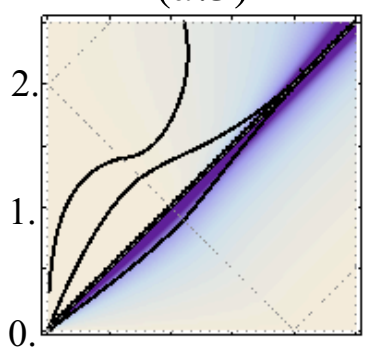

(a.4)

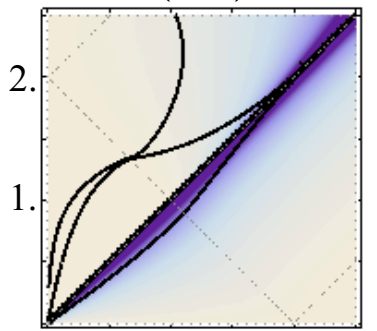

0. (b.1)

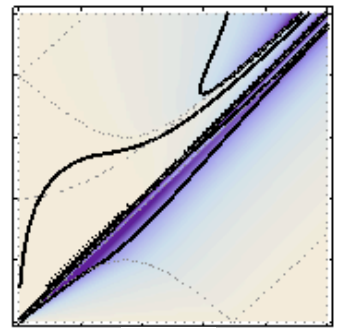

(b.2)

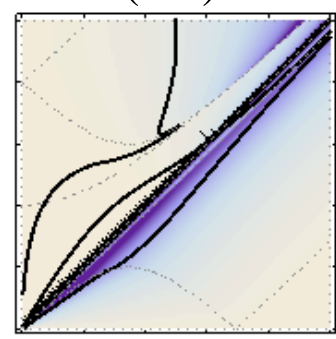

(b.3)

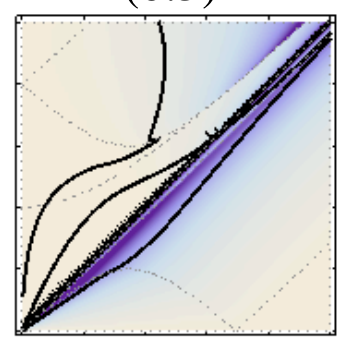

(b.4)

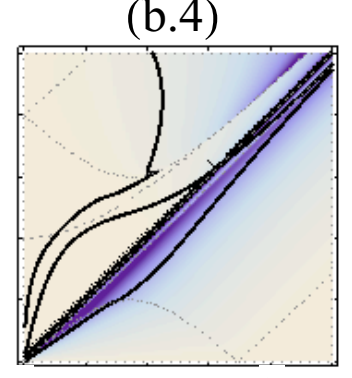

0.

$\mathrm{k} / k_{F}$ (c.1)

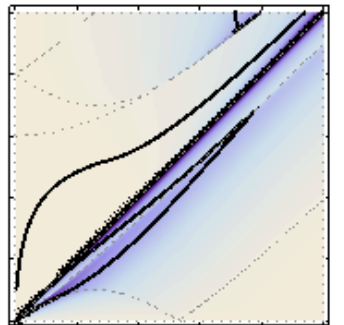

(c.2)

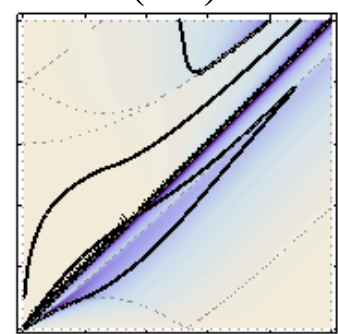

(c.3)

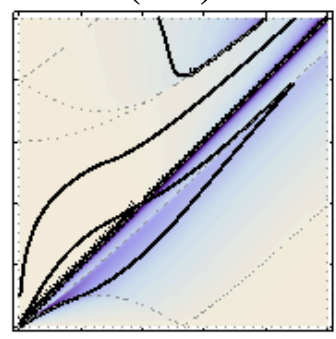

(c.4)

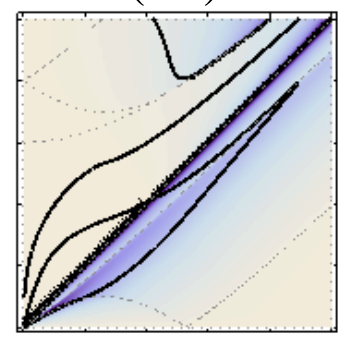

$0 . \quad 1.2$.

\section{$\frac{2}{3}$}

Figure 5: (Color online) Plasma excitations and damping (analogous to column (a) of [figure][3][]3 for graphene double layers. The difference with [figure][4][]4, is that the energy gap $E_{g}$ may not be the same for both layers. Columns (a, b, c) correspond to an induced gap $E_{g} / \mu=\{0.0,1.0,1.5\}$, respectively, only for one of the layers. Although the chemical potential is the same for both layers, a gap is not induced on the second layer (asymmetric case). Rows $(1,2,3,4)$ show plasmon branches for inter-layer separations $k_{F} d=\{0.1,0.5,1.0,5.0\}$. 
(a.1)

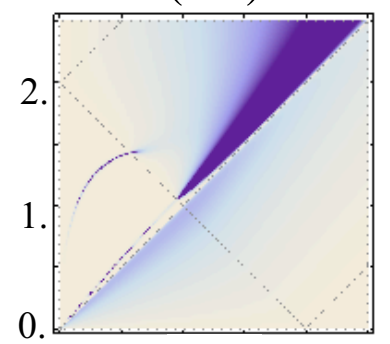

(a.2)

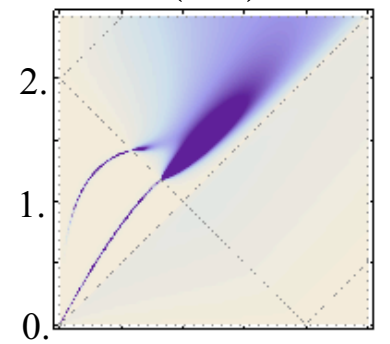

(a.3)

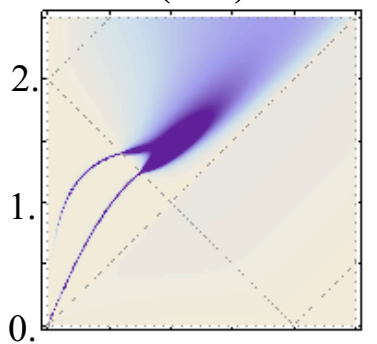

(a.4)

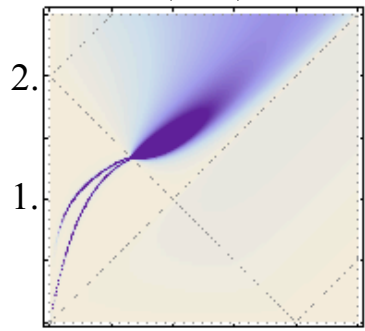

0 .

1. 2 . (b.1)

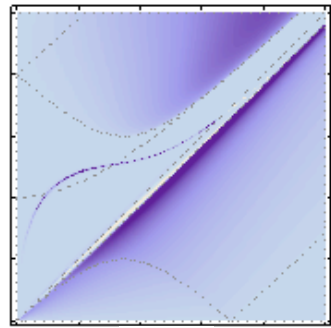

(b.2)

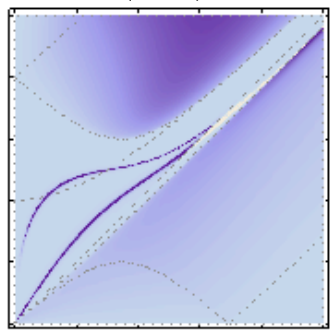

(b.3)

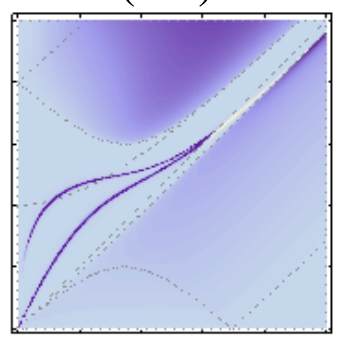

(b.4)

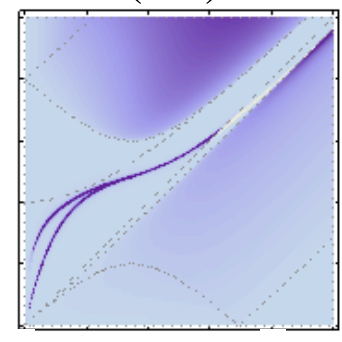

k $/ k_{F}$ (c.1)

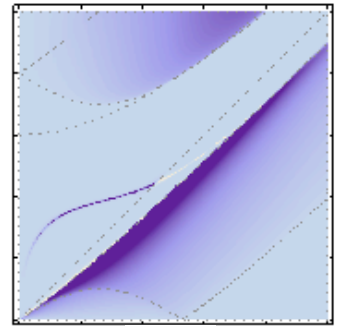

(c.2)

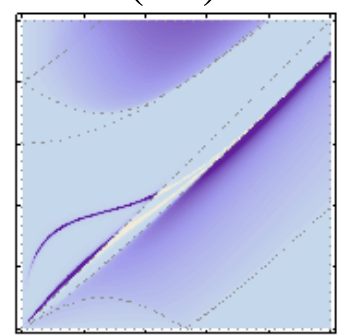

(c.3)

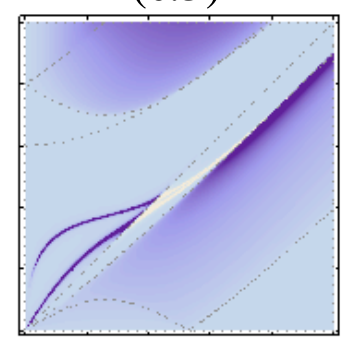

(c.4)

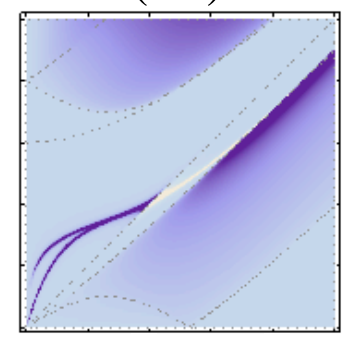

$0 . \quad 1 . \quad 2$.

Figure 6: (Color online) Plots similar to panels (b) in [figure][3][]3 showing $\Im m \Pi_{11}^{(0)}$ for double graphene layer configuration. For columns (a, b, c), we chose $E_{g} / \mu=\{0.0,1.0,1.5\}$, respectively. The induced gap and chemical potential are the same for both layers (symmetric case). Rows $(1,2,3,4)$ display plasmon branches for inter-layer distances $k_{F} d=\{0.1,0.5,1.0,5.0\}$. 
(a.1)

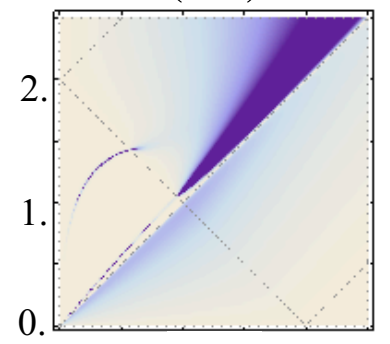

(a.2)

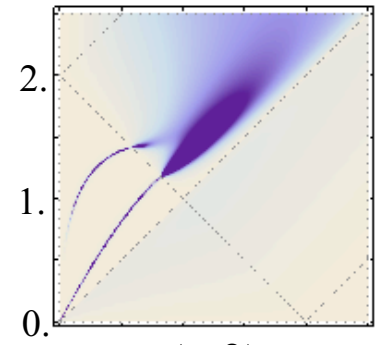

(a.3)

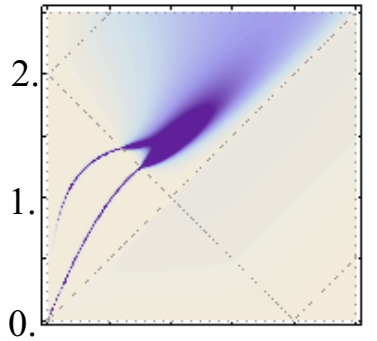

(a.4)

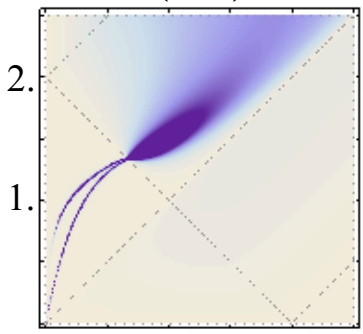

$0 . \quad 1 . \quad 2$. (b.1)

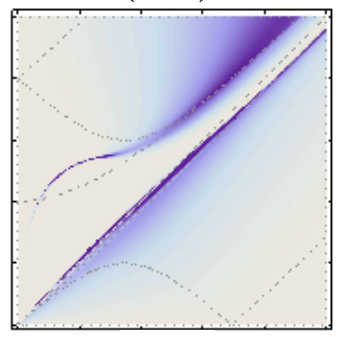

(b.2)

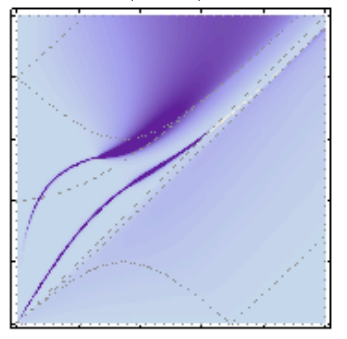

(b.3)

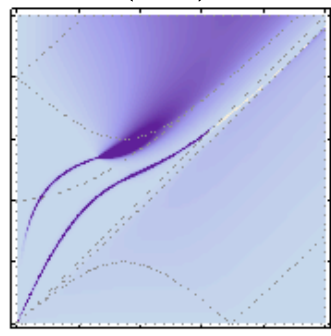

(b.4)

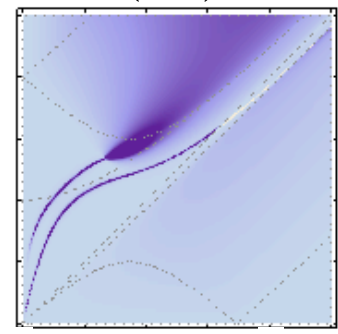

0 . (c.1)

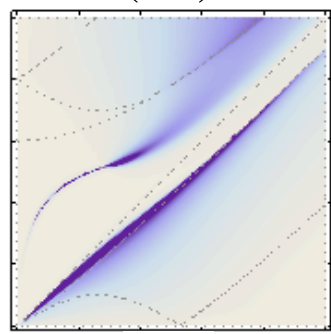

(c.2)

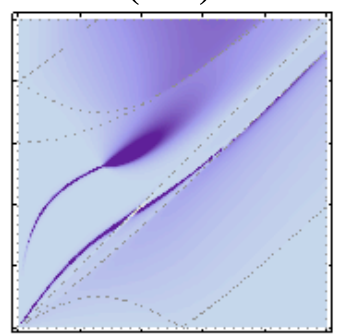

(c.3)

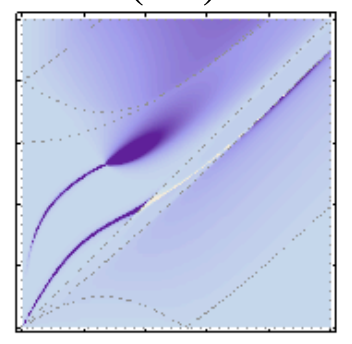

(c.4)

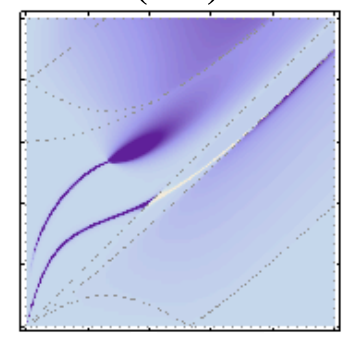

$0 . \quad 1 . \quad 2$.

.

$\frac{2}{3}$

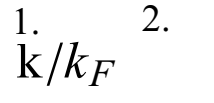

Figure 7: (Color online) Plots similar to those in column (b) of [figure][3][]3 showing $\mathfrak{I} m \Pi_{11}^{(0)}$ for a pair of graphene layers. Columns ( $\mathrm{a}, \mathrm{b}, \mathrm{c})$ were obtained using $E_{g} / \mu=\{0.0,1.0,1.5\}$, respectively. Although the chemical potential is uniform for both layers, a gap is not induced on the second layer (asymmetric case). Panels $(1,2,3,4)$ show plasmon branches for inter-layer distances $k_{F} d=$ $\{0.1,0.5,1.0,5.0\}$. 